This is a preprint or reprint of a paper intended for presentation at a conference. Because changes may be made before formal publication, this is made available with the understanding that it will not be cited or reproduced withoul the permission of the author.

\title{
PREDICTION OF THE ACOUSTIC FIELD ASSOCIATED WITH INSTABILITY WAVE SOURCE MODEL FOR A COMPRESSIBLE JET
}

\author{
V. Golubev ${ }^{*}$, R. Mankbadi ${ }^{\dagger}$ \\ Embry-Riddle Aeronautical University \\ M. Dahl ${ }^{\ddagger}$ \\ NASA Glenn Research Center
}

\begin{abstract}
This paper provides preliminary results of the study of the acoustic radiation from the source model representing spatially-growing instability waves in a round jet at high speeds. The source model is briefly discussed first followed by the analysis of the produced acoustic directivity pattern. Two integral surface techniques are discussed and compared for prediction of the jet acoustic radiation field.
\end{abstract}

\section{INTRODUCTION}

In [1], a model for the sound source in supersonic jets is developed based on the assumption that the unsteady fluctuations in the noise-producing jet mixing layer are dominated by coherent, large-scale structures which closely resemble instability waves [2], [3]. Within the integral energy approach, the coherent structure is modeled based on splitting the flow into three components: a time-average mean component, a largescale wave-like coherent component, and a fine-scale random turbulence component. The kinetic energy equations obtained from time and phase averaging of the full compressible Navier-Stokes equations can be integrated across the jet to produce a set of ordinary differential equations describing interactions among various scales of motion. With certain shape assumptions, these equations can be solved to yield the development of each flow component, which may be used as a source for subsequent acoustic radiation predictions.

In the present study, the source obtained in [1] is treated as the jet near field, and two integral methods based on the Kirchhoff surface approach [4], [5] are applied to investigate pattern of the jet acoustic radiation in the far

\footnotetext{
* Assistant Professor, Member of AIAA

$\dagger$ Professor and Chair, Associate Fellow of AIAA

$\ddagger$ Research Scientist, Member of AIAA
}

field. The corresponding numerical formulations are discussed, and the numerical results are compared against the analytical benchmark for the monopole source radiation. The comparison is then extended to the prediction of the acoustic radiation of the jet helical modes.

\section{JET ACOUSTIC SOURCE FORMULATION}

Following [1], we consider a high-Reynolds number turbulent jet issuing from a nozzle of diameter $D$ in a still air. The jet is assumed shock-free, and is excited by a single-frequency instability wave of Strouhal number $S t=f D / U$. The density and the velocities are normalized by the jet exit density and velocity at the centerline. The development of large-scale, coherent, wave-like structure profiles in the compressible round jet is then obtained using the integral energy method and following the locally-parallel linear stability theory.

For the purpose of the present analysis, the results are presented as single-frequency, single-azimuthal number modes which, for the pressure perturbation, take the form,

$$
p^{\prime}(x, r, \phi, t)=\hat{p}(r) A(x) \exp \left(i \int_{0}^{x} \alpha d \chi-i \omega t\right) \cos (n \phi)+c c,
$$

where ${ }^{\wedge}$ denotes the transversal shape function of the transversal coordinate $r$ at a given location along the jet (also, the eigenfunctions corresponding to a given $n$ and $\omega), \mathrm{n}$ is the azimuthal wave number indicating the rotation around the jet centerline, $\alpha$ is the axial wave number, $\omega$ is the excitation frequency, and $c c$ denotes the complex conjugate. $A(x)$ is the complex amplitude function of $x$ which is determined, in general, from a nonlinear analysis.

Figures 1 and 2 compare results obtained in [1] for the predicted development of the jet instability wave amplitude function using the linear and nonlinear 
analyses, for $S t=0.2, \mathrm{n}=1$, and the jet Mach numbers $M=0.9,1.2,1.5$, and 1.8. Figure 1 shows the development of the pressure wave magnitude along the jet axis at $r=D / 2$. Note that the nonlinear effects limit the amplitude growth and shift the peak amplitude towards the jet nozzle exit. These effects become more pronounced as the Mach number increases. Figure 2 illustrates the variation of the pressure magnitude in the radial direction at a fixed axial position corresponding to $x=1.5 D$.
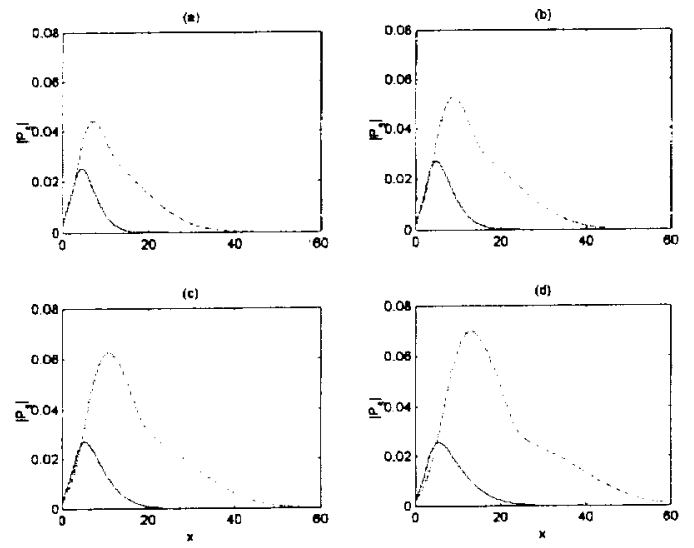

Figure 1. Axial variation of the pressure magnitude for the jet instability wave with linear (dashed line) and nonlinear (solid line) amplitude development: $r=D / 2$, $S t=0.2, \mathrm{n}=1$, (a) $M=0.9$, (b) $M=1.2$, (c) $M=1.5$, (d) $M=1.8$.
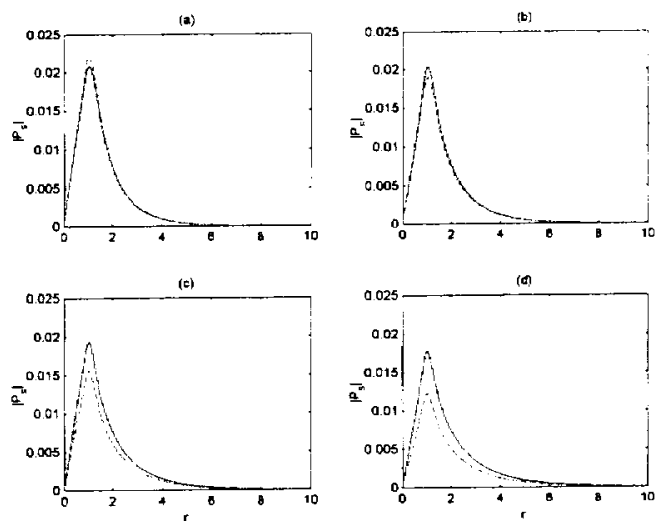

Figure 2. Radial variation of the pressure magnitude for the jet instability wave with linear (dashed line) and nonlinear (solid line) amplitude development: $x=1.5 \mathrm{D}$, $S t=0.2, \mathrm{n}=1$, (a) $M=0.9$, (b) $M=1.2$, (c) $M=1.5$, (d) $M=1.8$.

\section{FORMULATIONS FOR ACOUSTIC PREDICTION}

Using the near-field data as illustrated in Figures 1 and 2 , the present work calculates and compares the far field acoustic radiation of the jet instability wave using two integral numerical techniques, the Kirchhoff method and its extension, the Surface-Integral Formulation (SIF). Both methods are based on evaluating the near-field unsteady flow data on a control surface surrounding the nonlinear flow region, and propagating the unsteady flow information to the far field through the linear flow region. However, the numerical implementations contain some essential differences, as discussed below.

\section{The Kirchhoff Method}

Following [4], a formulation of the Kirchhoff method is implemented with assumption of a uniform flow outside the control surface $S$ which encloses all the sound sources. In the outer region, the convective wave equation for the acoustic pressure $p^{\prime}$ applies,

$$
\nabla^{2} p^{\prime}-\frac{1}{c_{0}^{2}}\left(U_{0} \frac{\partial}{\partial x}+\frac{\partial}{\partial t}\right)^{2} p^{\prime}=0
$$

where $c_{0}$ is the speed of sound, and $U_{0}$ is the free stream velocity in $x$-direction which is less than the speed of sound. The Prandtl-Glauert transformation

$$
x_{0}=x, \quad y_{0}=\beta y, \quad z_{0}=\beta z
$$

converts the convective wave equation into the simple wave equation which can be solved for the acoustic pressure field,

$p^{\prime}(X, Y, Z, t)=-\frac{1}{4 \pi} \int_{3}^{1} \frac{1}{R_{0}}\left[\frac{p^{\prime}}{R_{0}} \frac{\partial R_{n}}{\partial n_{0}}+\frac{\partial p}{\partial n_{0}}+\frac{1}{c_{o} \beta^{2}} \frac{\partial p}{\partial t} \frac{\partial}{\partial n_{0}}\left(R_{0}-M x_{0}\right)\right]_{\tau} d S_{0}$

where the subscript " $O$ " denotes the transformed variables, $\mathbf{n}=\left(\begin{array}{lll}n_{x} & n_{y} & n_{z}\end{array}\right)$ is the outward normal to the surface $S$, and the subscript $\tau$ indicates that all the values are calculated at the retarded time,

$$
t_{r}=t-\tau
$$

$R_{a}$ is the distance between the observer and the surface point in the Prandtl-Glauert coordinates,

$$
R_{o}=\left\{\left(X-x_{s}\right)^{2}+\beta^{2}\left[\left(Y-y_{s}\right)^{2}+\left(Z-z_{s}\right)^{2}\right]\right\}^{1 / 2}
$$

and $\tau$ and $\beta$ are defined as 


$$
\begin{gathered}
\tau=\frac{\left[R_{0}-M_{0}\left(X-x_{s}\right)\right]}{c_{o} \beta^{2}}, \\
\beta=\left(1-M_{0}{ }^{2}\right)^{\frac{1}{2}}
\end{gathered}
$$

where $M_{0}=U_{0} / c_{o}$ is the free stream Mach number.

Equation (4) describes the sound pressure at an observer point $(X, Y, Z)$ in terms of the source information provided on the control surface $S$. In general, the pressure and its normal and time derivatives are required to be specified through numerical simulation, or some other means. This may represent a difficulty using the Kirchhoff method as the numerical results tend to be very sensitive to the accuracy of the derivatives estimate.

\section{The Surface-Integral Formulation (SIF)}

An alternative Surface-Integral Formulation (SIF) has been developed in [5] to eliminate the need to estimate pressure derivatives on the control surface. In application to the present jet noise study, a control surface is considered as a cylinder of radius $a$ and length $L$ enclosing all the jet acoustic sources, as illustrated in Figure 3. It will be further assumed that the mean flow outside the cylinder is stagnant, and thus the acoustic disturbances are described by the simple wave equation in the cylindrical coordinates:

$$
\nabla^{2} p-\frac{1}{c^{2}} \frac{\partial^{2} p}{\partial t^{2}}=0
$$

Since the jet pressure modes (1) are excited with a specified frequency $\omega$, it is convenient to transform formulation to the frequency domain by applying the Fourier transform to (9). The integral solution is then obtained in the form,

$$
p(\mathbf{X}, \omega)=-\int\left[G \frac{\partial p}{\partial n}-p \frac{\partial G}{\partial n}\right] d S
$$

where $p(\mathbf{X}, \omega)$ is the acoustic pressure at the observation point $\mathbf{X}=(X, R, \Phi), G$ is the Green function, $\mathbf{n}$ is the normal to the surface $S$, and $p(\mathbf{x}, \omega)$ is the pressure distribution on the control surface at a point $\mathbf{x}=(x, r, \phi)$.

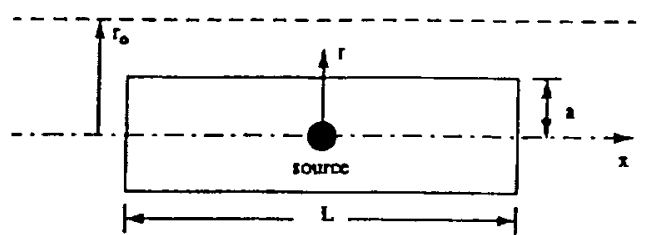

Figure 3. Cylinder used for validation of numerical codes.

The approach of the SIF formulation is to seek a solution such that $G=0$ on the control surface $r=a$, using the method of images. Morse and Ingard [6] give the Green function for emission from a cylinder of radius $a$ at a point $R$, for $R>a$, as

$$
\mathrm{g}_{1}=\Xi \int_{-\infty}^{\infty} e^{i k_{x}(X-x)} J_{m}(q r) H_{m}(q R) d k_{x}
$$

where

$$
\Xi=\frac{i}{8 \pi} \sum_{m=0}^{m=\infty} \varepsilon_{m} \cos [m(\phi-\Phi)]
$$

and

$$
\begin{array}{ll}
q=\sqrt{k^{2}-k_{x}^{2}} & \text { for } k_{x}<k \\
q=i \sqrt{k_{x}^{2}-k^{2}} & \text { for } k_{x}>k,
\end{array}
$$

where $\varepsilon_{m}$ is Neumann constant, $\varepsilon_{m}=1$ for $m=0$, and $\varepsilon_{m}$ $=2$ for $m>0$. $J_{m}$ is the $m$ th order Bessel function, $H_{m}$ is the $m$ th order Hankel function of the first kind, and $k_{x}$ is the wavenumber in the $x$-direction of the acoustic disturbance at the surface. For the image of the point $R$ at $a^{2} / R$, the Green function is

$$
\mathrm{g}_{2}=\Xi \int_{-\infty}^{\infty} e^{i k_{x}(X-x)} H_{m}(q r) J_{m}\left(q \frac{a^{2}}{R}\right) d k_{x}
$$

Adding the two solutions (11) and (15), one obtains, after algebraic manipulations, the required Green function which satisfies the control surface condition $G(r=a)=0$,

$$
\begin{aligned}
G=\Xi & \int_{-\infty}^{\infty} e^{i k_{x}(X-x)} \frac{H_{m}(q R)}{H_{m}(q a)} \times \\
& {\left[J_{m}(q r) H_{m}(q a)-J_{m}(q a) H_{m}(q r)\right] d k_{x} }
\end{aligned}
$$


and, using at $r=a$ the relationship

$$
H^{\prime}(u) J(u)-J^{\prime}(u) H(u)=\frac{2 i}{\pi u}
$$

one also obtains,

$$
\begin{aligned}
\frac{\partial G}{\partial r}=\frac{1}{4 \pi^{2} a} \sum_{m=0}^{m=\infty} & \varepsilon_{m} \cos [m(\phi-\Phi)] \times \\
& \int_{-\infty}^{\infty} e^{i k_{x}\left(x_{o}-x\right)} \frac{H_{m}(q R)}{H_{m}(q a)} d k_{x}
\end{aligned}
$$

With $G=0$ at the control surface, the integral solution reduces to

$$
p(X, R, \Phi)=\int p \frac{\partial G}{\partial r} a d x d \phi
$$

Substituting, the acoustic field is obtained as

$$
\begin{aligned}
& p(X, R, \Phi)=\frac{1}{4 \pi^{2}} \iint p(x, a, \phi) \sum_{m=0}^{m=\infty} \varepsilon_{m} \cos [m(\phi-\Phi)] \\
& \times \int_{-\infty}^{\infty} e^{i k_{x}(X-x)} \frac{H_{m}(q R)}{H_{m}(q a)} d k_{x} d x d \phi
\end{aligned}
$$

The formula (19) describes the relation between the acoustic far-field and the pressure distribution on a cylindrical control surface surrounding the jet noise sources. To perform the volume integration, the surface pressure on the cylindrical surface is taken as

$$
p(x, a, \phi, \omega)=\sum_{n=0}^{n=\infty} p_{n}(x, a, \omega) \cos n \phi
$$

and the integration over the azimuthal direction is then performed to obtain the final formula,

$$
\begin{gathered}
p(X, R, \Phi, \omega)= \\
\frac{1}{2 \pi} \sum_{m=0}^{m=\infty} \cos m \Phi \int_{0}^{L} p_{m}(x, a, \omega) \times \\
\int_{-k_{L}}^{k_{L}} e^{i k_{x}(X-x)} \frac{H_{m}(q R)}{H_{m}(q a)} d k_{x} d x
\end{gathered}
$$

where $k_{L}$ is selected as the Nyquist limit, $k_{L}=\pi / \Delta x$, and $\Delta x$ is spacing between the $x$-points on the cylinder. For the numerical implementation, the order of integration in (21) can be reversed.

\section{Point Source Comparison}

Prior to applying to the jet acoustic predictions, the two numerical codes implementing the Kirchhoff method and the SIF formulation are validated against the point source test.

The pressure distribution on the control cylindrical surface resulting from a monopole located in the middle of the cylinder, as shown in Figure 3, is described by

$$
p\left(\mathbf{X}_{s}, t\right)=\frac{1}{r_{s}} \operatorname{Re}\left[\exp \left\{-i \omega t_{r s}\right\}\right],
$$

where, similar to (5) and (6),

$$
r_{s}=\left\{\left(x_{s}-x^{\prime}\right)^{2}+\beta^{2}\left[\left(y_{s}-y^{\prime}\right)^{2}+\left(z_{s}-z^{\prime}\right)^{2}\right]\right\}^{1 / 2}
$$

$\left(x^{\prime}, y^{\prime}, z^{\prime}\right)$ is the source location at the midpoint of the centerline, and the retarded time $t_{r s}$ on the cylinder surface is determined from

$$
t_{r s}=t-\frac{r_{s}-M_{0}\left(x_{s}-x^{\prime}\right)}{c_{o} \beta^{2}}
$$

For calculations using the Kirchhoff method, both the pressure and its normal derivative to the surface have to be introduced in (4). The code takes into account the contribution from both the left and the right bases of the cylinder surrounding the point source, and thus the length $L$ can be taken as small as needed. For computations, the values of $a=1$ and $L / a=10$ are used. For further comparison, the obtained time-domain results are converted into frequency domain using MATLAB FFT. For the SIF numerical implementation, the frequency-domain analysis is carried out by first factoring out time $t$ in (22). The contribution from the bases is not currently taken into account, and the ratio $L / a$ should be large enough for accurate predictions. In all the computations, the number of points per wavelength in each direction is about 12 , which provides an error of less then $0.5 \%$ according to estimates in [4]. 


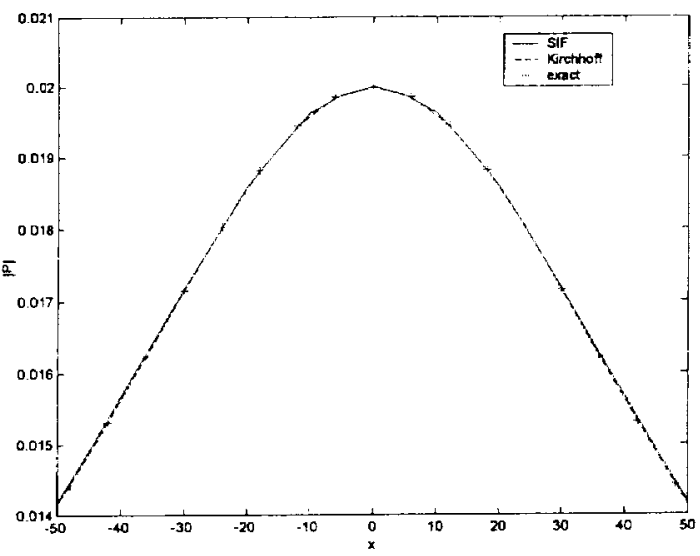

Figure 4. Validation against the point source.

The results of the two codes are compared in Figure 4 for $\omega / c_{0}=1$ and $M_{0}=0$. In the calculations, $a=1$, and the observer points are located at $R / a=50,-50<X / a<50$. Note that the two codes show very close results for the acoustic pressure amplitude, and both match with the exact solution. The codes are very fast and require only seconds of CPU time per observer point on the PC computer.

\section{RESULTS AND DISCUSSION}

The two formulations are now applied to predict the acoustic radiation pattern produced by the coherent cold jet structure described by (1). A single helical mode with $n=1$ is excited with Strouhal number $S t=0.2$, and the acoustic response is calculated for the jet Mach numbers $M=0.9,1.2,1.5$, and 1.8 . Figures 1 and 2 illustrate the development of the near-field unsteady pressure amplitude obtained from the linear and nonlinear analyses, as discussed above.

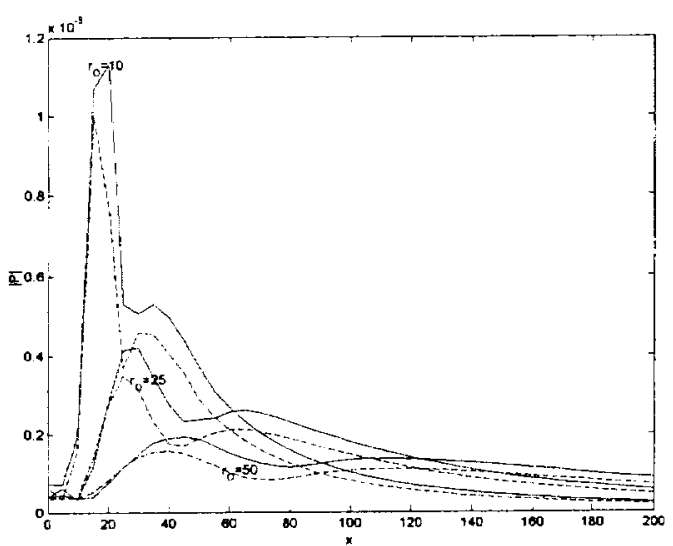

Figure 5. Pressure amplitude distribution along the $x$ axis at $r /(D / 2)=10,25,50$ : Kirchhoff method (solid line) and SIF (dashed line) predictions. Source data from linear jet instability wave analysis: $S t=0.2, \mathrm{n}=1$, $M=1.2$.

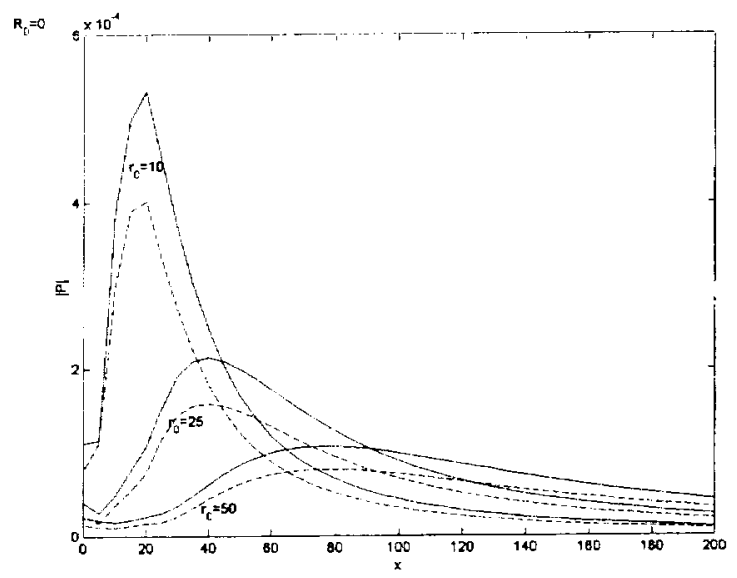

Figure 6. Pressure amplitude distribution along the $x$ axis at $r /(D / 2)=10,25$, 50: Kirchhoff method (solid line) and SIF (dashed line) predictions. Source data from nonlinear jet instability wave analysis: $S t=0.2$, $\mathrm{n}=1, M=1.2$.

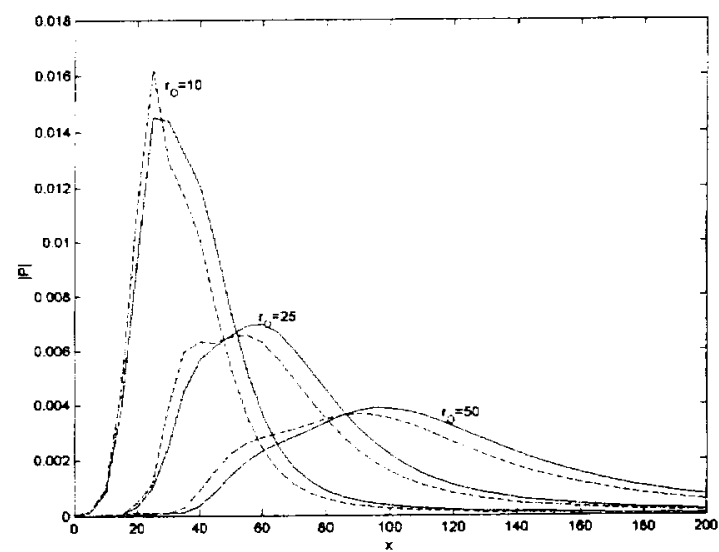

Figure 7. Pressure amplitude distribution along the $x$ axis at $r /(D / 2)=10,25,50$ : Kirchhoff method (solid line) and SIF (dashed line) predictions. Source data from linear jet instability wave analysis: $S t=0.2, \mathrm{n}=1$, $M=1.8$.

5

American Institute of Aeronautics and Astronautics 


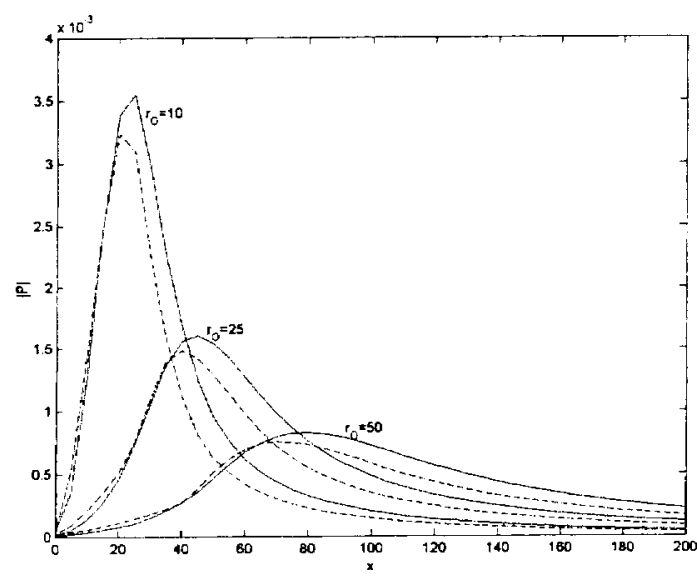

Figure 8. Pressure amplitude distribution along the $x$ axis at $r /(D / 2)=10,25,50$ : Kirchhoff method (solid line) and SIF (dashed line) predictions. Source data from nonlinear jet instability wave analysis: $S t=0.2$, $\mathrm{n}=1, M=1.8$.

Figures 5-8 present the comparison of the results from the two prediction codes for two cases, corresponding to $M=1.2$ and 1.8 , for both linear and nonlinear wave amplitude development. The observer points are located parallel to the jet axis (from the jet origin) at three radii, $r /(D / 2)=10,25$ and 50 , from the jet centerline. All the lengths in these and subsequent figures are normalized by the initial jet radius, $D / 2$. Note that the two codes show similar results, but a discrepancy in both the amplitude and the phase is also evident, and more pronounced at $M=1.2$. In all the computations, the right boundary of the control surface is taken far enough so that the sound source has practically decayed and no contribution from the base is expected. The actual values taken for $L /(D / 2)$ vary for different cases since, from Figure 1, the source extends much further in the linear cases. The differences between the results predicted by the two codes may be primarily attributed to the sensitivity of the Kirchhoff method to the accuracy of the normal pressure derivative estimates on the control surface. The normal derivatives are obtained by numerical approximation from the source data given at uniformly discretized radial locations. The latter itself is produced by interpolating from a non-uniform mesh used by the source prediction code. Moreover, the sensitivity study for the placement of the control surface has revealed that, while the value of the proper control surface radius is stable in the SIF code calculations for different cases, the Kirchhoff method code requires careful selection of the control surface location in each case. A typical range of the control surface radii has varied within $r_{s} /(D / 2)=1.5 \ldots 2.5$.
Below this limit, the source is not adequately enclosed by the control surface; and for larger values of $r_{s}$, the source has sufficiently decayed so the data input is not adequate for accurate acoustic predictions. In that case, as $r_{s}$ further increases, the results usually show decreasing values of the acoustic pressure amplitude at a fixed far-field observer location.
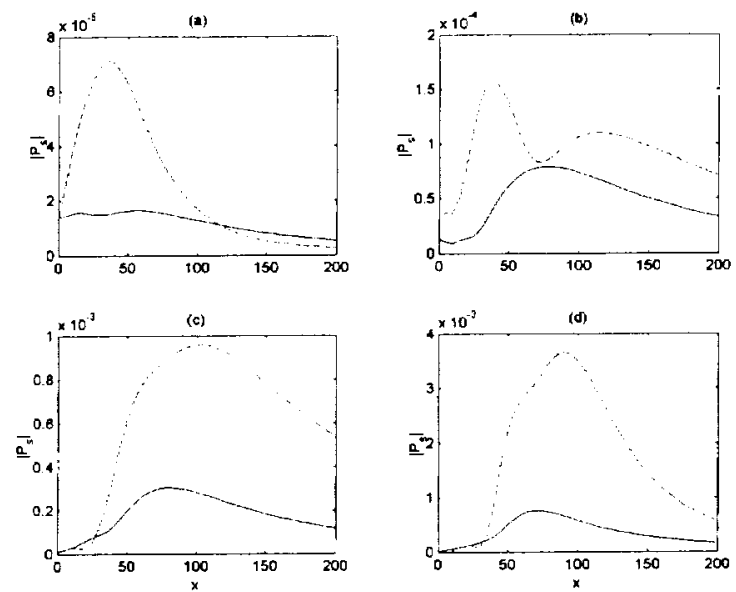

Figure 9. Pressure amplitude distribution along the $x$ axis at $r /(D / 2)=50$. Source data from linear (dashed line) and nonlinear (solid line) jet instability wave analysis, $S t=0.2, \mathrm{n}=1:$ (a) $M=0.9$, (b) $M=1.2$, (c) $M=1.5$, (d) $M=1.8$.

The rest of the results presented in Figures 9-11 are based on the SIF code calculations. Figure 9 summarizes the acoustic predictions for all the selected cases obtained at $r /(D / 2)=50$ along the jet axis. The results obtained for the linear and nonlinear source amplitude development are compared. As expected, the acoustic response is much higher in the linear cases, and both show the response increasing for higher jet Mach numbers.

Finally, Figures 10 and 11 show the sound pressure level (SPL) contours calculated for the linear and nonlinear wave amplitude development, respectively. The predictions for the linear source amplitude analysis show a trend to develop a multi-lobe directivity pattern, which points to the effect of the non-compact source distribution due to the excessively large axial extension of the instability wave structure. The nonlinear results exhibit the characteristic jet directivity pattern as observed in the experiment (compare with Figure 12 from [7], with the data obtained for $S t=0.2$ and $M=2.1$ ) 

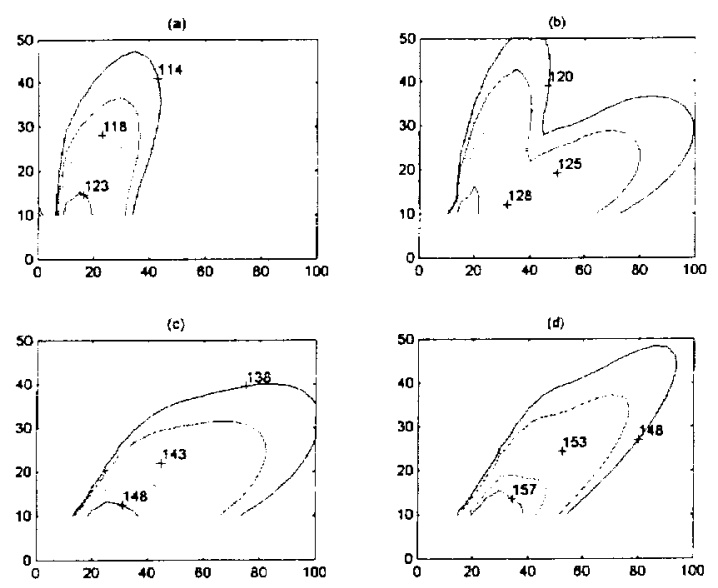

Figure 10. Predicted SPL contours for linear jet instability wave analysis, $S t=0.2, \mathrm{n}=1$ : (a) $M=0.9$, (b) $M=1.2$, (c) $M=1.5$, (d) $M=1.8$.
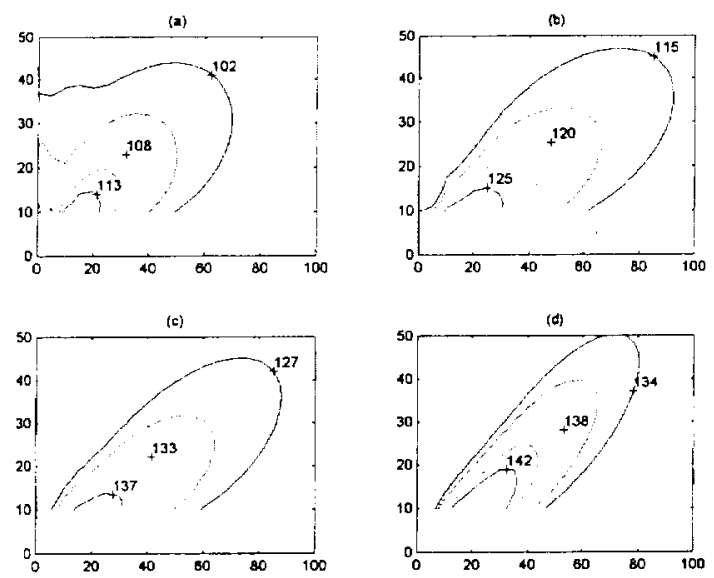

Figure 11. Predicted SPL contours for nonlinear jet instability wave analysis, $S t=0.2, \mathrm{n}=1$ : (a) $M=0.9$, (b) $M=1.2$, (c) $M=1.5$, (d) $M=1.8$.

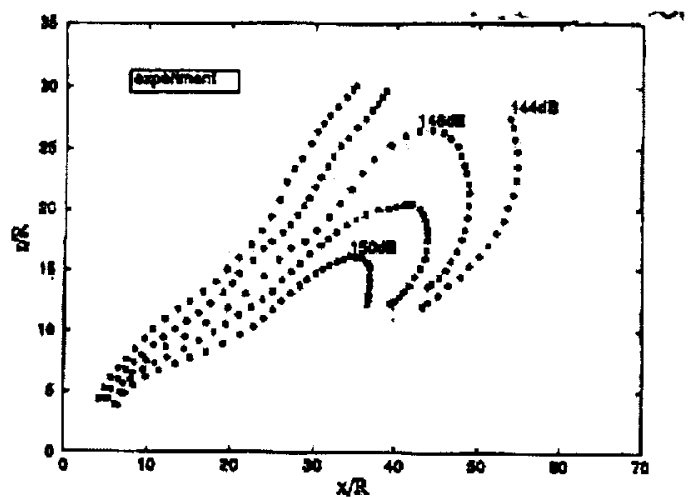

Figure 11. Measured jet acoustic directivity. Data from [7]: $S t=0.2, M=2.1$.

\section{CONCLUSIONS}

Two integral surface numerical techniques, the Kirchhoff method and the Surface-Integral Formulation, were compared for prediction of the acoustic radiation field produced by the source model representing spatially-growing instability waves in a round jet at high speeds [1]. Both methods require the unsteady pressure information on a control surface surrounding the sound source, and propagate this information to the far field through the linear flow region. In addition, the Kirchhoff method requires normal and time derivatives to be specified on the control surface, and is sensitive to the accuracy of their approximation.

The acoustic results based on the provided data for the linear and nonlinear amplitude development of the jet instability wave were compared. The acoustic response was found to be much higher in the linear cases, and results for both linear and nonlinear source analyses showed the acoustic response increasing for higher jet Mach numbers. The acoustic directivity predictions for the linear source analysis revealed a trend to develop a multi-lobe pattern attributed to the non-compact source distribution due to the large axial extension of the instability wave structure. The nonlinear source analysis produced a characteristic jet directivity pattern which is in qualitative agreement with observations.

\section{ACKNOWLEDGEMENTS}

Dr. Golubev would like to acknowledge the support of the Ohio Aerospace Institute in this work.

\section{REFERENCES}

1. Dahl, M.D. and Mankbadi, R.R., "Analysis of Three-Dimensional, Nonlinear Development of WaveLike Structure in a Compressible Jet," submitted for presentation at the AIAA Aeroacoustics Meeting, June 2002.

2. Tam, C.K.W. and Burton, D.E., "Sound Generated by Instability Waves of Supersonic Flows. Part 2. Axisymmetric Jets," Journal of Fluid Mechanics, Vol. 138 , pp.273-295, 1984.

3. Mankbadi, R.R., Hayder, M.E. and Povinelli, L.A., "Structure of Supersonic Jet Flow and Its Radiated Sound," AIAA Journal, Vol. 31, pp. 897-906, 1994.

4. Lyrintzis, A. and Mankbadi, R.R., "Prediction of the Far-Field Jet Noise Using Kirchhoff's 
Formulation,"AIAA Journal, Vol. 34, pp.413-416, 1996.

5. Mankbadi, R.R., Shih, S.-H., Hixon, D.R., Stuart, J.T. and Povinelli, L.A., "A Surface-Integral Formulation for Jet Noise Prediction Based on the Pressure Signal Alone," Journal of Computational Acoustics, Vol. 6, pp. 307-320, 1998.

6. Morse,P.M. and Ingard, K.U., "Theoretical Acoustics," Princeton Univ. Press, 1986.

7. Troutt, T.R. and McLaughlin, D.K., "Experiments on the Flow and Acoustic Properties of a Moderate Reynolds Number Supersonic Jet," Journal of Fluid Mechanics, Vol. 116, pp.123-156, 1982. 